\title{
Insatisfação corporal e comportamentos de risco para o desenvolvimento de transtornos alimentares: uma avaliação entre estudantes de medicina
}

Body dissatisfaction and risky behavior for the development of eating disorders: an evaluation among medical students

Insatisfacción corporal y conductas de riesgo para el desarrollo de trastornos alimentarios: una evaluación entre estudiantes de medicina

Leonardo Bruno Melo Reis

https://orcid.org/0000-0002-1571-0502

Georgia Guimarães de Castro - https://orcid.org/0000-0001-7274-8657

Larissa Rafaella Pereira Tôrres - https://orcid.org/0000-0003-1778-1600

Rodrigo Satoshi Oda Santos - https://orcid.org/0000-0003-0585-9175

João Vitor Costa - https://orcid.org/0000-0002-3062-9032

Matheus Gomes Pereira - https://orcid.org/0000-0001-9858-4734

Dorothéa Schmid França - https://orcid.org/0000-0002-4647-5218

\section{RESUMO:}

Introdução: Transtornos alimentares são condições complexas, graves e potencialmente fatais. Apresentam repercussões significativas nos âmbitos físico, psicológico, social e econômico. Suas complicações abrangem diferentes sistemas, variando desde disfunções nutricionais, ósseas, cardíacas, psiquiátricas e metabólicas. Objetivo: avaliar o grau de insatisfação corporal e comportamentos de risco para o desenvolvimento de transtornos alimentares (TAs) em estudantes de Medicina de uma instituição de ensino privada no Norte de Minas Gerais. Métodos: 0 presente estudo é uma avaliação transversal e observacional, na qual 106 acadêmicas de medicina responderam ao Body Shape Questionnaire (BSQ), ao Teste de Atitudes Alimentares (EAT-26) e a um questionário sociodemográfico para análise do perfil sociodemográfico das estudantes, bem como a presença de condição de risco de transtornos alimentares nas 
mesmas. Resultados: A média de idade foi de 22,1 anos. O IMC médio foi de $21,86 \mathrm{~kg} / \mathrm{m}^{2}$, com desvio-padrão de $3,23 \mathrm{~kg} / \mathrm{m} 2$. A maioria das estudantes $(87,7 \%)$ estavam em eutrofia. De acordo com o EAT-26, $42,5 \%$ das entrevistadas estavam em risco de desenvolvimento de TA. Segundo o BSQ, 39,6\% relataram algum grau de insatisfação corporal. O risco de desenvolvimento de distúrbios da alimentação mostrou-se maior naquelas que relataram uma insatisfação moderada (100\%) a grave $(66,7 \%)$. Também se observou maior risco naquelas com sobrepeso (60\%) e obesidade $(66,7 \%)$, se comparadas ao grupo eutrófico $(39,8 \%) .72 \%$ das entrevistadas referiram medo de engordar, e 76\% mostraram-se, em alguma frequência, adeptas a dietas de emagrecimento. Portanto, a amostra avaliada apresentou elevados índices de insatisfação corporal, bem como risco elevado de desenvolvimento de TA. Além disso, verificouse grande adesão a hábitos dietéticos. Conclusão: Portanto, a amostra avaliada apresenta níveis elevados de insatisfação corporal, bem como maior risco para desenvolvimento de TA, bem como uma grande adesão a hábitos dietéticos.

Palavras chave: Comportamento alimentar, satisfação pessoal, estudantes universitárias, insatisfação corporal, transtornos alimentares.

\section{ABSTRACT:}

Introduction: Eating disorders are complex, serious and potentially fatal conditions. They present significant repercussions in the physical, psychological, social and economic spheres. Its complications cover different systems, ranging from nutritional, bone, cardiac, psychiatric and metabolic dysfunctions. Objective: to evaluate the degree of body dissatisfaction and risky behavior for the development of eating disorders (EDs) in medical students from a private educational institution in the north of Minas Gerais. Methods: The present study is a cross-sectional and observational assessment, in which 106 medical students answered the Body Shape Questionnaire (BSQ), the Eating Attitudes Test (EAT-26) and a sociodemographic questionnaire to analyze the sociodemographic profile of the students, as well as the presence of the risk conditions of similar disorders in them. Results: The average age was 22.1 years. The average BMI was $21.86 \mathrm{~kg} / \mathrm{m}^{2}$, with a standard deviation of $3.23 \mathrm{~kg} / \mathrm{m}^{2}$. Most of the students $(87.7 \%)$ were eutrophic. According to EAT-26, 42.5\% of respondents were at risk of developing ED. According to BSQ, 39.6\% reported some degree of body dissatisfaction. The risk of developing eating

2 Debates em Psiquiatria, Rio de Janeiro, 2021; 11:1-27

https://doi.org/10.25118/2763-9037.2021.v11.217 
disorders was higher in those who reported moderate $(100 \%)$ to severe dissatisfaction $(66.7 \%)$. A higher risk was also observed in those with overweight $(60 \%)$ and obesity $(66.7 \%)$, when compared to the eutrophic group (39.8\%). $72 \%$ of the interviewees reported fear of gaining weight, and $76 \%$ were, at some frequency, adept at slimming diets. Therefore, an evaluated sample offers high levels of body dissatisfaction, as well as a high risk of developing ED. In addition, there was great adherence to dietary habits. Conclusions: Therefore, the evaluated sample presents high levels of body dissatisfaction, as well as a higher risk of developing ED, as well as a great adherence to dietary habits.

\section{Keywords:}

Feeding behavior, personal satisfaction, university students, body dissatisfaction, eating desorders.

\section{RESUMEN:}

Introducción: Los trastornos alimentarios son condiciones complejas, graves y potencialmente fatales. Presentan repercusiones significativas en los ámbitos físico, psicológico, social y económico. Sus complicaciones abarcan diferentes sistemas, que van desde disfunciones nutricionales, óseas, cardíacas, psiquiátricas y metabólicas. Objetivo: evaluar el grado de insatisfacción corporal y conductas de riesgo para el desarrollo de trastornos alimentarios (TCA) en estudiantes de medicina de una institución educativa privada en el norte de Minas Gerais. Métodos: Este estudio es una evaluación observacional transversal, en la que 106 estudiantes de medicina respondieron el Body Shape Questionnaire (BSQ), el Eating Attitudes Test (EAT-26) y un cuestionario sociodemográfico para analizar el perfil sociodemográfico de los estudiantes. como la presencia de una condición de riesgo de trastornos alimentarios en ellos.

Resultados: La edad promedio fue de 22,1 años. El IMC promedio fue de $21,86 \mathrm{~kg} / \mathrm{m}^{2}$, con una desviación estándar de 3,23 kg / m2. La mayoría de los estudiantes $(87,7 \%)$ eran eutróficos. Según el EAT-26, el 42,5\% de los encuestados estaban en riesgo de desarrollar TCA. Según el BSQ, el $39,6 \%$ informó algún grado de insatisfacción corporal. El riesgo de desarrollar trastornos alimentarios fue mayor en aquellos que informaron una insatisfacción moderada (100\%) a grave $(66,7 \%)$. También se observó un mayor riesgo en aquellos con sobrepeso $(60 \%)$ y obesidad $(66,7 \%)$, en 
comparación con el grupo eutrófico (39,8\%). El 72\% de los entrevistados informaron tener miedo a aumentar de peso y el $76 \%$ eran, con cierta frecuencia, adeptos a las dietas de adelgazamiento. Por tanto, la muestra evaluada mostró altos niveles de insatisfacción corporal, así como un alto riesgo de desarrollar DE. Además, hubo una gran adherencia a los hábitos alimentarios. Conclusiones: Por tanto, la muestra evaluada presenta altos niveles de insatisfacción corporal, así como un mayor riesgo de desarrollar DE, así como una gran adherencia a los hábitos alimentarios.

Palabras clave: Conducta alimentaria, satisfacción personal, estudiantes universitarios, insatisfacción corporal, transtornos de la alimentacion.

Como citar: Reis LBM, Castro GG, Tôrres LRP, Santos RSO, Costa JV, Pereira MG, França DS. Insatisfação corporal e comportamentos de risco para o desenvolvimento de transtornos alimentares: uma avaliação entre estudantes de medicina. Debates em Psiquiatria, Rio de Janeiro, 2021; 11:1-27. https://doi.org/10.25118/2763-9037.2021.v11.217

\section{Conflito de interesses: declaram não haver}

Fonte de financiamento: declaram não haver

Parecer CEP: Faculdades Integradas Pitágoras de Montes Claros, MG (Parecer 4.251.794).

Recebido em: 11/10/2021

Aprovado em: 01/12/2021

Publicado em: 30/12/2021

\section{Introdução}

Os transtornos alimentares (TAs) são condições complexas, graves e potencialmente fatais, que apresentam repercussões significativas nos âmbitos físico, psicológico, social e econômico [1]]. Suas complicações podem ser diversas e abrangem diferentes sistemas, variando desde disfunções nutricionais, ósseas, cardíacas, psiquiátricas e metabólicas [2] ]. Dentre os principais TAs, destacam-se a anorexia nervosa, a bulimia nervosa e o transtorno da compulsão alimentar (TCA) [ㅍ-ㄴ]. 
Estudos epidemiológicos acerca dos TAs demonstram resultados variáveis, dependendo da amostra e dos métodos de avaliação. Entretanto, estimase que o TCA (Transtorno da Compulsão Alimentar) é o transtorno mais prevalente, superando a Bulimia Nervosa, podendo atingir de 0,8\% a 1,9\% da população, a depender do país [ㅌ]. Pesquisas apontam que até $30 \%$ das mulheres jovens adultas apresentam algum tipo de padrão alimentar de risco - como prática de dietas restritivas, não necessariamente enquadrado em um diagnóstico $[\underline{1}, \underline{5}]$.

Além disso, sabe-se que a prevalência dos transtornos alimentares, antes mais significativa em países ocidentais e industrializados, está em ascensão mundial, principalmente devido às modificações culturais e nos meios de comunicação [ㅁ]. Dessa forma, tem-se que os mesmos são, atualmente, uma problemática global em saúde.

A cultura ocidental, ao pregar uma extrema valorização da magreza, gera uma preocupação excessiva com o corpo e um medo patológico do aumento de peso. Dessa forma, os indivíduos tornam-se mais propensos a desenvolver transtornos alimentares, na tentativa de adequar-se ao padrão imposto. Estudos apontam que a pressão social incide principalmente sobre mulheres jovens. Logo, essas são mais propensas ao desenvolvimento de comportamentos de risco para transtornos alimentares (CRTA) []]. Atualmente, ainda é referida na literatura uma maior prevalência de TAs em estudantes de cursos de graduação na área da saúde. Isso ocorre, pois, nesses, a boa forma física e a aparência padrão estão ainda mais relacionados ao sucesso profissional []].

Os principais instrumentos para avaliação dos CRTA podem ser divididos em três categorias: avaliação clínica, automonitorização e questionários autoaplicáveis. O último, dentre esses, se destaca como importante método de triagem, devido à praticidade e eficiência na avaliação de um grande número de pessoas, além de serem menos onerosos ao sistema de saúde [ㅁ].

Devido à importância epidemiológica e ao grande impacto na saúde pública, torna-se necessário ampliar as formas de rastreamento do comportamento alimentar de risco, visando o diagnóstico precoce dos transtornos alimentares. Nesse contexto, o presente estudo tem como objetivo avaliar o grau de insatisfação corporal e comportamentos de risco 
para o desenvolvimento de transtornos alimentares em estudantes de Medicina.

\section{Metodologia}

O presente estudo configura-se como uma avaliação transversal e observacional, realizado com universitárias da graduação em medicina de uma instituição privada no município de Montes Claros, Minas Gerais. A pesquisa foi aprovada pelo Comitê de Ética das Faculdades Integradas Pitágoras de Montes Claros, segundo o parecer 4.251.794. Acadêmicas do $1^{0}$ ao $8^{\circ}$ período, devidamente matriculadas no curso de medicina, foram convidadas a participar do estudo. Foram excluídos os homens e mulheres com idade inferior a 18 anos.

As estudantes preencheram um formulário sociodemográfico, com idade e estado civil, além de informarem peso e estatura. Também responderam a questionários auto preenchíveis e validados em língua portuguesa, o Teste de Atitudes Alimentares (Eating Attitudes Test/EAT-26) e o Questionário de Imagem Corporal (Body Shape Questionnaire/BSQ).

Foi utilizado para análise de dados o software SPSS versão 25 , realizando teste qui-quadrado para comparar a frequência de comportamento de risco para transtornos alimentares entre os distintos grupos de estudantes, diferenciadas por meio das variáveis sociodemográficas, perfil de peso e classificação do BSQ. Considerou-se como EAT-26 negativo as alunas com baixo e nenhum risco para TA; EAT-26 positivo, as alunas classificadas com moderado e alto risco para TA.

Com relação ao Body Shape Questionnaire, considerou-se BSQ negativo as universitárias que não tiveram ou apresentavam insatisfação leve com a sua imagem corporal, e BSQ positivo, as estudantes que apresentaram insatisfação moderada e grave.

O Teste de Atitudes Alimentares (Eating Attitudes Test/EAT-26) tem como finalidade avaliar o risco de desenvolvimento de transtornos alimentares, e é composto por 26 questões, cujas respostas podem ser "sempre", "muito frequentemente", "frequentemente", "às vezes", "raramente" e "nunca", onde a primeira equivale a 3 pontos, a segunda a 2 pontos, a terceira a 1 ponto e as três últimas iguais a 0 pontos, exceto pela questão 25 , que é pontuada em escala inversa [10]. 
Os itens são divididos em três grupos: o primeiro consiste em evitar alimentos que engordam e preocupação em emagrecer. O segundo é composto por afirmações acerca de pensamentos sobre comida $e$ referentes à bulimia. No terceiro e último consta itens relativos ao autocontrole ao alimentar e a pressão exercida por terceiros para ganho ponderal [11].

As participantes que obtiveram pontuação de 0 a 9 , foram consideradas isentas de risco; com pontuação de 10 a 20 foram consideradas de baixo risco; aquelas com pontuação maior que 20 pontos foram consideradas com padrão alimentar anormal [10]. [스exo 1]

Já o Questionário de Imagem Corporal (Body Shape Questionnaire/BSQ), [Anexo 2], tem como fim a auto avaliação da imagem corporal, integrando 34 questões que abordam hábitos dietéticos, receio com ganho de peso e principalmente índices de satisfação com o próprio corpo. Cada uma apresenta pontuações de 1 a 6 , que gradua a periodicidade de "nunca" a "sempre" acerca da aparência nas últimas quatro semanas [12].

Para as participantes com pontuação menor ou igual a 110 pontos foi considerada como um padrão de normalidade e tido como ausência de insatisfação com a imagem corporal. Resultados entre 110 e 138 pontos foram classificados como insatisfação leve; entre 139 e 167 como insatisfação moderada; e acima de 167 pontos como insatisfação grave com a imagem corporal [12]. [Anexo 2]

Com relação ao estado civil, a existência de cônjuge foi questionada e o tipo foi classificado em: solteira - pessoa que tenha o estado civil solteira e sem companheiro; casada; divorciada - pessoa que tenha estado civil, homologado por decisão judicial, de divorciada; união estável há mais de seis meses - em caso de relação, duradoura e registrada em cartório, de convivência com companheiro(a).

As idades das participantes foram agrupadas em três faixas etárias: até 21 anos, de 22 a 25 anos e acima de 25 anos.

Os questionários foram identificados apenas com o primeiro nome da participante, sendo aplicados por meio da plataforma Google Forms, dos dias 20/10/2020 a 25/10/2020. Os dados foram tabulados pela equipe de estatística. Após concordância com o TCLE (Termo de Consentimento Livre 
e Esclarecido) [Anexo 3], pelas participantes selecionadas, os questionários foram aplicados.

O EAT-26 e o BSQ têm suas propriedades psicométricas comprovadas para o grupo estudado. Além de estrutura fatorial satisfatória, os instrumentos correlacionaram-se com as medidas de avaliação da insatisfação corporal e comportamento alimentar, discriminando diferentes estratos de IMC, como também comprovaram sua consistência interna e reprodutibilidade.

O peso e a estatura foram autorreferidos e o índice de massa corporal (IMC) foi calculado pela equação de Quetelet (IMC $=$ Peso/Altura ${ }^{2}$ ) e interpretado conforme a Organização Mundial de Saúde (OMS): IMC menor $18,5 \mathrm{~kg} / \mathrm{m}^{2}=$ abaixo do peso, entre $18,5 \mathrm{~kg} / \mathrm{m}^{2}$ e $24,9 \mathrm{~kg} / \mathrm{m}^{2}=$ peso normal, entre $25 \mathrm{~kg} / \mathrm{m}^{2}$ e $29,9 \mathrm{~kg} / \mathrm{m}^{2}$ = sobrepeso, igual ou acima de 30 $\mathrm{kg} / \mathrm{m}^{2}=$ obesidade [13$]$.

Os dados antropométricos não são exatos por estes serem informados pelas próprias participantes, porém, esse modelo de obtenção de dados é extensamente empregado em estudos científicos em função da correspondência equivalente entre as informações relatadas e os valores diretamente aferidos [14].

\section{Resultados}

Participaram do estudo 106 das 288 acadêmicas regularmente matriculadas do $1^{\circ}$ ao $8^{\circ}$ período do curso de medicina. A pesquisa revelou uma média de idade de 22,1 anos e um desvio-padrão de 3,3 anos na população estudada.

Em relação ao estado civil, $95,2 \%$ se declararam solteiras, e 4,8\% casadas/em união estável/divorciadas.

As medidas antropométricas autorreferidas apontaram média de peso de $58,8 \mathrm{~kg}$ e um desvio-padrão de $9,1 \mathrm{~kg}$, uma altura média de $1,64 \mathrm{~m}$ com desvio-padrão de $0,05 \mathrm{~m}$. Quanto ao IMC, a média foi de $21,86 \mathrm{~kg} / \mathrm{m}^{2} \mathrm{com}$ desvio-padrão de $3,23 \mathrm{~kg} / \mathrm{m} 2$.

Em relação à referida classificação, $87,7 \%$ das estudantes encontravamse em eutrofia, 9,4\% em sobrepeso, e 2,9\% em algum grau de obesidade. A tabela 1 evidencia o perfil sociodemográfico obtido. 
Tabela 1 - Perfil sociodemográfico das estudantes

\begin{tabular}{|c|c|c|c|c|}
\hline \multicolumn{2}{|l|}{ Variáveis } & $\mathrm{N}$ & $\%$ & Valor de $p$ \\
\hline \multirow[t]{6}{*}{ Faixa Etária } & & & & 0,000 \\
\hline & Até 21 anos & 45 & 42,9 & \\
\hline & De 22 a 25 & 52 & 49,5 & \\
\hline & anos & & & \\
\hline & Acima de 25 & 8 & 7,6 & \\
\hline & anos & & & \\
\hline \multicolumn{2}{|c|}{ Índice de Massa Corporal (IMC) } & & & 0,000 \\
\hline & Normal & 93 & 87,7 & \\
\hline & Sobrepeso & 10 & 9,4 & \\
\hline & Obesidade & 3 & 2,9 & \\
\hline \multirow[t]{6}{*}{ Estado Civil } & & & & 0,000 \\
\hline & Solteira & 99 & 95,2 & \\
\hline & União & 5 & 4,8 & \\
\hline & Estável/ & & & \\
\hline & Casada/ & & & \\
\hline & Divorciada & & & \\
\hline
\end{tabular}

Quando questionadas sobre a possibilidade de aumento de peso, $72 \%$ das acadêmicas referiram algum medo de engordar. Dessas, $79 \%$ são eutróficas. Além disso, $67 \%$ das entrevistadas mostraram-se preocupadas com o desejo de ser magra.

Quanto à avaliação de risco para o desenvolvimento de transtornos alimentares, baseada no preenchimento do Teste de Atitudes Alimentares, obteve-se que $42,5 \%$ das entrevistadas estão em condição de risco.

Os resultados do EAT-26 foram comparados com os dados do questionário sociodemográfico e do Body Shape Questionnaire, como evidencia a Tabela 2. 
Tabela 2 - Prevalência das estudantes em relação ao risco de transtorno alimentar

\begin{tabular}{|c|c|c|c|c|c|c|}
\hline \multirow{3}{*}{ Variáveis } & \multirow{3}{*}{$\begin{array}{l}\text { Amostra } \\
\mathrm{n}(\%)\end{array}$} & \multicolumn{4}{|c|}{$\begin{array}{l}\text { Riscos para Transtornos } \\
\text { Alimentares (EAT-26) }\end{array}$} & \multirow[b]{3}{*}{$p \#$} \\
\hline & & \multicolumn{2}{|c|}{ Não } & \multicolumn{2}{|c|}{ Sim } & \\
\hline & & $\mathrm{N}$ & $\%$ & $\mathrm{n}$ & $\%$ & \\
\hline \multirow[t]{2}{*}{ Total } & 106 & 61 & 57,5 & 4 & 42,5 & \\
\hline & $(100,0)$ & & & 5 & & \\
\hline \multirow[t]{2}{*}{ Faixa Etária } & & & & & & 0,91 \\
\hline & & & & & & 4 \\
\hline \multirow[t]{2}{*}{ Até 21 anos } & $45(42,9)$ & 26 & 57,8 & 1 & 42,2 & \\
\hline & & & & 9 & & \\
\hline \multirow[t]{2}{*}{ De 22 a 25 anos } & $52(49,5)$ & 30 & 57,7 & 2 & 42,3 & \\
\hline & & & & 2 & & \\
\hline Acima de 25 anos & $8(7,6)$ & 4 & 50,0 & 4 & 50,0 & \\
\hline \multirow[t]{2}{*}{ Índice de Massa Corporal (IMC) } & & & & & & 0,32 \\
\hline & & & & & & 4 \\
\hline \multirow[t]{2}{*}{ Normal } & $93(87,7)$ & 56 & 60,2 & 3 & 39,8 & \\
\hline & & & & 7 & & \\
\hline Sobrepeso & $10(9,4)$ & 4 & 40,0 & 6 & 60,0 & \\
\hline Obesidade & $3(2,8)$ & 1 & 33,3 & 2 & 66,7 & \\
\hline \multirow[t]{2}{*}{ Estado Civil } & & & & & & 0,08 \\
\hline & & & & & & 0 \\
\hline \multirow[t]{2}{*}{ Solteira } & $99(95,2)$ & 59 & 59,6 & 4 & 40,4 & \\
\hline & & & & 0 & & \\
\hline União Estável/Casada/Divorciada & $5(4,8)$ & 1 & 20,0 & 4 & 80,0 & \\
\hline \multirow[t]{2}{*}{ Body Shape Questionnaire } & & & & & & 0,00 \\
\hline & & & & & & 0 \\
\hline \multirow[t]{2}{*}{ Nenhuma } & $64(60,4)$ & 49 & 76,6 & 1 & 23,4 & \\
\hline & & & & 5 & & \\
\hline \multirow[t]{2}{*}{ Leve } & $32(30,2)$ & 11 & 34,4 & 2 & 65,6 & \\
\hline & & & & 1 & & \\
\hline \multirow[t]{2}{*}{ Moderada } & $7(6,6)$ & 0 & 0,0 & 7 & 100 & \\
\hline & & & & & 0 & \\
\hline Grave & $3(2,8)$ & 1 & 33,3 & 2 & 66,7 & \\
\hline
\end{tabular}

n:frequência absoluta; \%: frequência relativa

Observou-se um maior risco relativo nas estudantes com sobrepeso $(60 \%)$ e obesidade $(66,7 \%)$, se comparadas ao grupo eutrófico $(39,8 \%)$.

Em relação à faixa etária, o grupo mais vulnerável está acima dos 25 anos $(50 \%)$. Foi verificado um maior risco relativo $(80 \%)$ entre as mulheres 10 Debates em Psiquiatria, Rio de Janeiro, 2021; 11:1-27 https://doi.org/10.25118/2763-9037.2021.v11.217 
casadas/em união estável/divorciadas, quando comparadas ao grupo de solteiras $(40,4 \%)$.

Em relação ao grau de insatisfação corporal, avaliado pelo BSQ, os resultados mostraram altos índices de positividade. Os escores variaram entre o mínimo de 34 e o máximo de 189 pontos e constatou-se que 39,6\% das estudantes têm algum grau de insatisfação. A positividade do EAT-26 mostrou-se maior nas estudantes que relataram uma insatisfação moderada $(100 \%)$ a grave $(66,7 \%)$ com a própria imagem.

Além disso, mesmo as entrevistadas que não relataram nenhum grau significativo de insatisfação corporal $(60,4 \%)$ no BSQ, também estão expostas ao risco de desenvolvimento de transtornos do comportamento alimentar $(23,4 \%)$, de acordo com os hábitos relatados no EAT-26. Não houveram participantes com dados capazes de preencher critérios para diagnóstico de um transtorno alimentar específico.

\section{Discussão}

Os transtornos do comportamento alimentar tiveram sua incidência mundial praticamente dobrada nos últimos 20 anos, denotando a crescente preocupação da população com sua imagem corporal [15]. O presente estudo utilizou dois questionários auto preenchíveis, validados em língua portuguesa, para avaliar o grau de insatisfação corporal e o risco de desenvolvimento de transtornos alimentares em jovens universitárias.

Encontrou-se na população estudada uma alta frequência de comportamentos disfuncionais. Apesar de o BSQ e o EAT-26 não realizarem diagnóstico, são meios eficazes de rastreamento e de avaliação de CRTA [16]. Logo, essa pesquisa indica um alto risco de desenvolvimento de TA no grupo avaliado.

No que diz respeito ao peso corporal, observou-se que $87,7 \%$ das entrevistadas eram eutróficas. Entretanto, mesmo na faixa de peso normal, ainda verificou-se um risco elevado $(39,8 \%)$ de desenvolvimento de distúrbios alimentares. Os referidos dados estão em concordância com outros estudos realizados em amostras semelhantes [의, 17-18].

Ao comparar as relações entre IMC e o risco de desenvolvimento de TA, observou-se maior vulnerabilidade nas estudantes com sobrepeso $(60 \%)$ e obesidade $(66,7 \%)$, se comparadas ao grupo eutrófico $(39,8 \%)$. 
De forma semelhante, Bosi [19] , ao analisar 189 estudantes universitárias, concluiu que a prevalência de possíveis distúrbios nutricionais era maior entre as alunas com sobrepeso; e que tanto a insatisfação com o peso corporal como a classificação do IMC estão diretamente associados a maiores pontuações no BSQ.

Resultados semelhantes foram encontrados por outros pesquisadores ao avaliarem amostras similares [20-23]. Esses resultados são alarmantes pois além das estudantes acima do peso grande parte das eutróficas se mostraram insatisfeitas com o próprio corpo, o que aponta uma tendência das universitárias em querer alcançar um padrão corporal baseado na magreza. Vale ressaltar que, diversas vezes, na busca pela estética ideal, são adotados padrões alimentares disfuncionais que podem contribuir para o desenvolvimento de doenças [24].

Em relação à imagem corporal, os resultados do BSQ indicam que 39,6\% das estudantes se mostraram insatisfeitas com a autoimagem. Além disso, $23,4 \%$ das entrevistadas que não relataram nenhum grau de desagrado ainda estão em risco de desenvolvimento de TA mediante a avaliação comportamental do EAT-26. Quando comparados a outros estudos, os resultados foram similares àqueles mais prevalentes na literatura: estudantes universitárias da área da saúde apresentam risco elevado para o desenvolvimento de transtornos alimentares [18].

Encontrou-se menores níveis de insatisfação que o importante estudo realizado por Alvarenga et al. [25] que avaliou o grau de satisfação com a autoimagem de 2.402 universitárias nas cinco regiões do país, e concluiu que $78,3 \%$ apresentaram algum grau de descontentamento, sendo que $64,4 \%$ desejam ser mais magras e apenas $21,7 \%$ se mostraram satisfeitas com corpo atual. Entretanto, no referido estudo, o instrumento de autoavaliação utilizado foi a Escala de Silhuetas de Stunkard. Os resultados aqui obtidos são semelhantes a outras pesquisas, nas quais o BSQ foi utilizado para avaliação [트-27].

Em uma revisão integrativa da literatura que comparava os graus de descontentamento com a imagem corporal obtidos em diversos estudos, constatou-se que a insatisfação foi maior quando avaliada por meio de escalas de silhuetas do que quando avaliada pelo BSQ. As primeiras avaliam somente o tamanho e a forma do corpo, enquanto o questionário 
aborda, também, pensamentos e comportamentos individuais, fornecendo uma triagem mais sensível [28].

Segundo a avaliação pelo EAT-26, 42,5\% das entrevistadas estão em risco de desenvolvimento de TA. O elevado índice de positividade corrobora com a premissa presente na literatura de que as estudantes da área da saúde são mais susceptíveis aos distúrbios do comportamento alimentar do que universitárias de outras áreas [르. Isso ocorre devido à forte associação entre a magreza e uma melhor imagem profissional.

Ao realizar um estudo com 127 acadêmicas entre 18 e 22 anos, Laus [30] comparou o risco de desenvolvimento de TA entre as mesmas, através da aplicação do EAT-26. A positividade foi significativamente maior nas estudantes da saúde (50\% em Nutrição; 24\% em Educação Física), em comparação às de humanas (13\% em Publicidade e Propaganda; $18 \%$ em Administração de Empresas).

A elevada associação entre a insatisfação com a imagem corporal e o risco de desenvolvimento de transtornos identificada nesta pesquisa corrobora com dados já publicados na literatura. No estudo de Alves et al. [31], foi constatado que o descontentamento com a autoimagem (BSQ+) era o maior fator de risco para a manifestação de comportamentos alimentares disfuncionais. Resultados semelhantes foram encontrados por outros pesquisadores [32-35].

Ao detalhar os hábitos, $51,88 \%$ relataram evitar o consumo de alimentos que contenham açúcar, e 55,66\% evitam o consumo de pratos ricos em carboidratos, como massas e pães. Além disso, 46,22\% referiram prestar atenção à quantidade de calorias ingeridas diariamente.

Um estudo realizado por Griegg et al. [36] evidenciou que, em um grupo de garotas entre 14 e 16 anos, insatisfeita com a sua imagem corporal, cerca de $57 \%(n=853)$ faziam dietas consideráveis não saudáveis e $36 \%$ faziam uso de laxantes, diuréticos, pílulas anorexígenas, além de realizar dietas extremamente restritivas. Desse modo, é notória a relação entre insatisfação corporal como grande fator de risco para o desenvolvimento de transtornos alimentares.

Em relação às limitações do estudo, destaca-se o fato de a aplicação dos testes ter sido realizada via plataformas digitais, em conformidade com as normas de isolamento social preconizadas durante a pandemia pelo 
Coronavírus (COVID-19). Questionários preenchidos por 42 indivíduos foram invalidados e retirados da análise estatística, reduzindo parcialmente a amostra. Desses, $38(90,48 \%)$ devido ao envio de dados incompletos/inconclusivos, e $4(9,52 \%)$ por terem sido preenchidos por homens.

Somado a isso, ressalta-se que questionários preenchíveis estão sujeitos à interpretação subjetiva das perguntas, o que pode interferir nos resultados. Ademais, tem-se a limitação dos dados antropométricos autorreferidos, o que reduz a precisão das informações. Entretanto, temse na literatura que dados como peso, altura e IMC autorreferidos têm sido amplamente utilizados em pesquisas científicas devido à elevada concordância entre os dados informados e aqueles precisamente medidos $[\underline{13}]$.

\section{Conclusão}

Os resultados do presente estudo demonstraram que a amostra avaliada apresentou elevados índices de insatisfação corporal, bem como risco elevado de desenvolvimento de transtornos alimentares. Além disso, verificou-se grande adesão a hábitos dietéticos. Os referidos achados denotam a alta vulnerabilidade das acadêmicas da área da saúde à pressão estética imposta pela sociedade. Os elevados índices encontrados denotam a necessidade da implementação de programas de educação e estratégias de intervenção direcionados a essa população de risco.

\section{Agradecimentos}

Agradecemos à instituição UNIFIPMOC por nos fornecer toda a base e recursos necessários para idealização e realização do trabalho, bem como a todas as acadêmicas que participaram do estudo e se dispuseram a responder os questionários disponibilizados. 


\section{Referências}

1. Rowe, E. Early detection of eating disorders in general practice. Australian Family Physician. 2017 Nov; 46(11), p.833. https://www.racgp.org.au/afp/2017/november/early-detection-ofeating-disorders/

2. Mitchell-Gieleghem A, Mittelstaedt ME., Bulik CM. Eating disorders and childbearing: Concealment and consequences. Birth. 2002 Sep;29(3):182-91. https://doi.org/10.1046/j.1523536X.2002.00186.X - PMid: 12153649

3. Cordás TA. Transtornos alimentares: classificação e diagnóstico. Rev. Psiquiatr. Clín. 2004 Jul;31(4):154-157. https://doi.org/10.1590/S0101-60832004000400003

4. American Psychiatric Association. (2013). Diagnostic and Statistical Manual of Mental Disorders. In Arlington (5th ed.). American Psychiatric Publishing. https://doi.org/10.1176/appi.books.9780890425596.744053

5. Kessler RC, Berglund PA, Chiu WT, Deitz AC, Hudson JI, Shahly V, Aguilar-Gaxiola S, Alonso J, Angermeyer MC, Benjet C, Bruffaerts R, Girolamo G., De Graaf R, De Haro JM, Kovess-Masfety V, Neill SO, Posada-Villa J, Sasu C, Scott K, Xavier M. (2013). The Prevalence and Correlates of Binge Eating. Biol Psychiatry, 73, 904-914. https://doi.org/10.1016/j.biopsych.2012.11.020 PMid:23290497 - PMCid:PMC3628997

6. Pinzon V, Nogueira FC. Epidemiologia, curso e evolução dos transtornos alimentares. Archives of Clinical Psychiatry. 2004 Jan;31(4):158-60. https://doi.org/10.1590/S0101$\underline{60832004000400004}$

7. Souto S, Ferro-Bucher JS. Práticas indiscriminadas de dietas de emagrecimento e o desenvolvimento de transtornos alimentares. Revista de Nutrição. 2006 Dec;19(6):693-704. https://doi.org/10.1590/S1415-52732006000600006 
8. Kessler AL, Poll FA. Relação entre imagem corporal, atitudes para transtornos alimentares e estado nutricional em universitárias da área da saúde. Jornal Brasileiro de Psiquiatria. 2018 Jun;67(2):11825. https://doi.org/10.1590/0047-2085000000194

9. Freitas S, Gorenstein C, Appolinario JC. Instrumentos para a avaliação dos transtornos alimentares. Jornal Brasileiro de Psiquiatria. 2002 Dec;24:34-8. https://doi.org/10.1590/S151644462002000700008

10. Bighetti F, Santos CB, Santos JE, Ribeiro RPP. Tradução e validação do Eating Attitudes Test em adolescentes do sexo feminino de Ribeirão Preto-SP. Jornal Brasileiro de Psiquiatria. 2004 Nov; 53(6):339-346. https://pesquisa.bvsalud.org/portal/resource/pt/lil-403090

11. Garner DM, Olmsted MP, Boher Y, Garfinkel PE. The Eating Attitudes Test: psychometric features and clinical correlates. Psychological Medicine. 1982; 12: 871-8. https://doi.org/10.1017/s0033291700049163 - PMid:6961471

12. Di Pietro M, Silveira DX. Internal validity, dimensionality and performance of the Body Shape Questionnaire in a group of Brazilian college students. Rev Bras Psiquiatr. 2009 Mar;31(1):21-4. https://doi.org/10.1590/S1516-44462008005000017 PMid: 19030718

13. World Health Organization (WHO). Obesity: preventing and managing the global epidemic. Geneva: WHO; 2000. https://apps.who.int/iris/handle/10665/42330

14. Reis JA, Silva Júnior CR, Pinho LD. Fatores associados ao risco de transtornos alimentares entre acadêmicos da área de saúde. Revista Gaúcha de Enfermagem. 2014 Jun;35(2):73-8. https://doi.org/10.1590/1983-1447.2014.02.42441 PMid:25158464

15. Uzunian LG, Vitalle MS. Habilidades sociais: fator de proteção contra transtornos alimentares em adolescentes. Ciência \& Saúde 
Coletiva. 2015 Fev;20:3495-508. https://doi.org/10.1590/1413812320152011.18362014 - PMid:26602727

16. Ferreira JES, Veiga GV. Comportamentos sugestivos de transtornos alimentares na adolescência: aspectos conceituais. Adolescência e Saúde. 2010 Jul;7(3):33-7.

https://doi.org/10.1590/0102-311X00192913 - PMid:25493997

17. Maia RG, Fiorio BC, Almeida JZ, Silva FR. Estado nutricional e transtornos do comportamento alimentar entre estudantes do curso de graduação em nutrição no Instituto Federal de Educação, Ciências e Tecnologia, Ceará, Brasil. Demetra: Alimentação, Nutrição \& Saúde. 2018 May 21;13(1):135-45. https://doi.org/10.1590/S1413-81232012001200024 PMid:23175415

18. Bandeira YE, Mendes AL, Cavalcante AC, Arruda SP. Avaliação da imagem corporal de estudantes do curso de Nutrição de um centro universitário particular de Fortaleza. Jornal Brasileiro de Psiquiatria. 2016 Jun;65(2):168-73. https://doi.org/10.1590/0047$\underline{2085000000119}$

19. Bosi ML, Luiz RR, Uchimura KY, Oliveira FP. Comportamento alimentar e imagem corporal entre estudantes de educação física. Jornal Brasileiro de Psiquiatria. 2008 Jan;57(1):28-33. https://doi.org/10.1590/S0047-20852008000100006

20. Miranda VP, Filgueiras JF, Neves CM, Teixeira PC, Ferreira ME. Insatisfação corporal em universitários de diferentes áreas de conhecimento. Jornal Brasileiro de Psiquiatria. 2012 Jan;61(1):2532. https://doi.org/10.1590/S0047-20852012000100006

21. Silva JD, Silva AB, Oliveira AV, Nemer AS. Influência do estado nutricional no risco para transtornos alimentares em estudantes de nutrição. Ciência \& Saúde Coletiva. 2012 Jan;17:3399-406. https://doi.org/10.1590/S1413-81232012001200024 PMid:23175415

22. Kakeshita IS, Almeida SD. Relação entre índice de massa corporal e a percepção da auto-imagem em universitários. Revista de Saúde Pública. 2006 Jun;40(3):497-504. 
https://doi.org/10.1590/S0034-89102006000300019 PMid: 16810375

23. Pinto DCD, Quadrado RP. Imagens em construção: satisfação corporal e transtornos alimentares em acadêmicos da área da saúde. Revista Latinoamericana de Estudos em Cultura y Sociedad. 2018 Fev; 759(4). https://doi.org/10.23899/relacult.v4i0.759

24. Morgan CM, Vecchiatti IR, Negrão AB. Etiologia dos transtornos alimentares: aspectos biológicos, psicológicos e socio-culturais. Jornal Brasileiro de Psiquiatria. 2002 Dec;24:18-23. https://doi.org/10.1590/S1516-44462002000700005

25. Alvarenga MD, Philippi ST, Lourenço BH, Sato PD, Scagliusi FB. Insatisfação com a imagem corporal em universitárias brasileiras. Jornal Brasileiro de Psiquiatria. 2010 Mar;59(1):44-51. https://doi.org/10.1590/S0047-20852010000100007

26. Fortes LS, Cipriani FM, Coelho FD, Paes ST, Ferreira ME. A autoestima afeta a insatisfação corporal em adolescentes do sexo feminino?. Revista Paulista de Pediatria. 2014 Sep;32(3):236-40. https://doi.org/10.1590/0103-0582201432314 - PMid:25479855 PMCid:PMC4227346

27. Garcia L, Milagres OG, Mourão L, Assis M, Palma A. Autopercepção da imagem corporal em estudantes de Educação Física e Medicina. Revista Brasileira de Atividade Física \& Saúde. 2011 Ago;16(1):25-30. https://doi.org/10.12820/rbafs.v.16n1p25-30

28. Souza AC, Alvarenga MD. Insatisfação com a imagem corporal em estudantes universitários: Uma revisão integrativa. Jornal Brasileiro de Psiquiatria. 2016 Jul;65(3):286-99. https://doi.org/10.1590/0047-2085000000134

29. Fiates GM, Salles RK. Fatores de risco para o desenvolvimento de distúrbios alimentares: um estudo em universitárias. Revista de Nutrição. 2001;14:3-6. https://doi.org/10.1590/S1415$\underline{52732001000400001}$ 
30. Laus MF, Moreira RD, Costa TM. Diferenças na percepção da imagem corporal, no comportamento alimentar e no estado nutricional de universitárias das áreas de saúde e humanas. Revista de Psiquiatria do Rio Grande do Sul. 2009 Dec;31(3):192-6. https://doi.org/10.1590/S0101-81082009000300009

31. Alves E, Vasconcelos FD, Calvo MC, Neves JD. Prevalência de sintomas de anorexia nervosa e insatisfação com a imagem corporal em adolescentes do sexo feminino do Município de Florianópolis, Santa Catarina, Brasil. Cadernos de Saúde Pública. 2008;24:503-12. https://doi.org/10.1590/S0102-311X2008000300004 PMid: 18327438

32. Damasceno ML, Schubert A, Oliveira AP, Sonoo CN, Vieira JL, Vieira LF. Associação entre comportamento alimentar, imagem corporal e esquemas de gênero do autoconceito de universitárias praticantes de atividades físicas. Revista Brasileira de Atividade Física \& Saúde. 2011 Fev;16(2):138-43. https://doi.org/10.12820/rbafs.v.16n2p138-143

33. Costa LD, Vasconcelos FD. Influência de fatores socioeconômicos, comportamentais e nutricionais na insatisfação com a imagem corporal de universitárias em Florianópolis, SC. Revista Brasileira de Epidemiologia. 2010 Set;13:665-76. https://doi.org/10.1590/S1415-790X2010000400011 PMid:21180855

34. Neto APV, Assis BP, Pinto JSF, Lucinda LMF, Silva MHS, Carmo RL, Damasceno VO. Avaliação da satisfação com a imagem corporal e uso de medicamentos anorexígenos e anabolizantes em estudantes universitários. Revista Interdisciplinar de Estudos Experimentais. $2018 \mathrm{Jul}$; 10: 15-23. https://doi.org/10.34019/2177-3459.2018.v10.27455

35. Wang SB, Haynos AF, Wall MM, Chen C, Eisenberg ME, Neumark-Sztainer D. Fifteen-Year Prevalence, Trajectories, and Predictors of Body Dissatisfaction From Adolescence to Middle Adulthood. Clin Psychol Sci. 2019;7(6):1403-1415. https://doi.org/10.1177/2167702619859331 - PMid:32864198 PMCid:PMC7451946 
36. Grigg M, Bowman J, Redman S. Disordered eating and unhealthy weight reduction practices among adolescent females. Prev Med 1996; 25:748-56. https://doi.org/10.1006/pmed.1996.0115 PMid: 8936578 


\section{Anexo 1 - Eating Attitudes Test (EAT-26)}

Opções de resposta: SEMPRE - MUITAS VEZES - AS VEZES - POUCAS VEZES - QUASE NUNCA - NUNCA

1. Fico apavorada com a ideia de estar engordando. SEMPRE // MUITAS VEZES // AS VEZES // POUCAS VEZES // QUASE NUNCA // NUNCA

2. Evito comer quando estou com fome. SEMPRE // MUITAS VEZES // AS VEZES // POUCAS VEZES // QUASE NUNCA // NUNCA

3. Sinto-me preocupada com os alimentos. SEMPRE // MUITAS VEZES // AS VEZES // POUCAS VEZES // QUASE NUNCA // NUNCA

4. Continuar a comer em exagero faz com que eu sinta que não sou capaz de parar. SEMPRE // MUITAS VEZES // AS VEZES // POUCAS VEZES // QUASE NUNCA // NUNCA

5. Corto os meus alimentos em pequenos pedaços. SEMPRE // MUITAS VEZES // AS VEZES // POUCAS VEZES // QUASE NUNCA // NUNCA

6. Presto atenção às quantidades de calorias dos alimentos que eu como. (ex. pães, batatas, arroz, etc.) SEMPRE // MUITAS VEZES // AS VEZES // POUCAS VEZES // QUASE NUNCA // NUNCA

7. Evito, particularmente, os alimentos ricos em carboidratos. (ex. pães, batatas, arroz, etc.) SEMPRE // MUITAS VEZES // AS VEZES // POUCAS VEZES // QUASE NUNCA // NUNCA

8. Sinto que os outros gostariam que eu comesse mais. SEMPRE // MUITAS VEZES // AS VEZES // POUCAS VEZES // QUASE NUNCA // NUNCA

9. Vomito depois de comer. SEMPRE // MUITAS VEZES // AS VEZES // POUCAS VEZES // QUASE NUNCA // NUNCA

10. Sinto-me extremamente culpada depois de comer. SEMPRE // MUITAS VEZES // AS VEZES // POUCAS VEZES // QUASE NUNCA // NUNCA

11. Preocupo-me com o desejo de ser mais magra. SEMPRE // MUITAS VEZES // AS VEZES // POUCAS VEZES // QUASE NUNCA // NUNCA

12. Penso em queimar calorias a mais quando me exercito. SEMPRE // MUITAS VEZES // AS VEZES // POUCAS VEZES // QUASE NUNCA // NUNCA

13. As pessoas me acham muito magra. SEMPRE // MUITAS VEZES // AS VEZES // POUCAS VEZES // QUASE NUNCA // NUNCA

14. Preocupo-me com a ideia de haver gordura em meu corpo. SEMPRE // MUITAS VEZES // AS VEZES // POUCAS VEZES // QUASE NUNCA // NUNCA

15. Demoro mais tempo para fazer as refeições do que as outras pessoas. SEMPRE // MUITAS VEZES // AS VEZES // POUCAS VEZES // QUASE NUNCA // NUNCA 
16. Evito comer alimentos que contenham açúcar. SEMPRE // MUITAS VEZES // AS VEZES // POUCAS VEZES // QUASE NUNCA // NUNCA

17. Costumo comer alimentos dietéticos. SEMPRE // MUITAS VEZES // AS VEZES // POUCAS VEZES // QUASE NUNCA // NUNCA

18. Sinto que os alimentos controlam minha vida. SEMPRE // MUITAS VEZES // AS VEZES // POUCAS VEZES // QUASE NUNCA // NUNCA

19. Demonstro autocontrole diante dos alimentos. SEMPRE // MUITAS VEZES // AS VEZES // POUCAS VEZES // QUASE NUNCA // NUNCA

20. Sinto que os outros me pressionam para comer. SEMPRE // MUITAS VEZES // AS VEZES // POUCAS VEZES // QUASE NUNCA // NUNCA

21. Passo muito tempo pensando em comer. SEMPRE // MUITAS VEZES // AS VEZES // POUCAS VEZES // QUASE NUNCA // NUNCA

22. Sinto desconforto após comer doces. SEMPRE // MUITAS VEZES // AS VEZES // POUCAS VEZES // QUASE NUNCA // NUNCA

23. Faço regimes para emagrecer. SEMPRE // MUITAS VEZES // AS VEZES // POUCAS VEZES // QUASE NUNCA // NUNCA

24. Gosto de sentir o meu estomago vazio. SEMPRE // MUITAS VEZES // AS VEZES // POUCAS VEZES // QUASE NUNCA // NUNCA

25. Gosto de experimentar novos alimentos ricos em calorias. SEMPRE // MUITAS VEZES // AS VEZES // POUCAS VEZES // QUASE NUNCA // NUNCA

26. Sinto vontade de vomitar após as refeições. SEMPRE // MUITAS VEZES // AS VEZES // POUCAS VEZES // QUASE NUNCA // NUNCA 


\section{Anexo 2 - Body Shape Questionnaire (BSQ)}

Opções de resposta: 1=Nunca, 2=Raramente, 3=Às vezes, 4=Frequentemente, 5=Muito Frequentemente, $6=$ Sempre

1. Ter-se sentido entediado(a) fez com você se passasse a preocupar com a sua forma física? 123456

2. Tem estado tão preocupado(a) com a forma do seu corpo que começou a pensar que deveria fazer dieta? 123456

3. Já Ihe ocorreu que as suas coxas, quadril/ancas ou nádegas são grandes demais em relação ao resto do seu corpo? 123456

4. Tem sentido medo de ficar gordo(a) ou mais gordo(a)? 123456

5. Preocupou-se com o seu corpo não ser firme o suficiente? 123456

6. Sentir-se cheio(a) (por exemplo, depois de ingerir uma refeição grande) fez com que se sentisse gordo(a)? 123456

7. Sentiu-se tão mal com a forma do seu corpo a ponto de chorar? 123456

8. Evitou correr por achar que seu corpo poderia balançar? 123456

9. Estar com pessoas magras, do mesmo sexo que o seu, faz com que se sinta desconfortável com a forma do seu corpo? 123456

10. Preocupou-se com que as suas coxas poderem ocupar muito espaço quando se senta? 123456

11. Comer, mesmo que uma pequena quantidade de comida, fez com que se sentisse gordo(a)? 123456

12. Tem reparado na forma do corpo de outras pessoas do mesmo sexo que o seu e, ao comparar-se, sentiu-se em desvantagem? 123456

13. Pensar na forma do seu corpo interferiu na sua capacidade de se concentrar noutras atividades (como por exemplo, ver televisão, ler ou acompanhar uma conversa)? 123456

14. Estar nu(nua), por exemplo, durante o banho, fez com que se sentisse gordo(a)? 123456

15. Já evitou usar roupas que o(a) façam reparar mais na forma do seu corpo? 123 456

16. Já imaginou remover (cortar) partes carnudas do seu corpo? 123456 
17. Comer doces, bolos e outros alimentos ricos em calorias fez com que se sentisse gordo(a)? 123456

18. Deixou de ir a eventos sociais (como por exemplo, festas) por sentir-se mal com a forma do seu corpo? 123456

19. Sentiu-se excessivamente grande e arredondado(a)? 123456 20. Sentiu vergonha do seu corpo? 123456

21. A preocupação com a forma do seu corpo levou-o(a) a fazer dieta? 123456

22. Sentiu-se mais contente em relação à forma do seu corpo quando seu estômago estava vazio (por exemplo, pela manhã)? 123456

23. Acredita que a forma do seu corpo se deve à sua falta de auto-controle(o)? 123 456

24. Preocupou-se com que outras pessoas vissem dobras na região da sua cintura ou estômago? 123456

25. Pensou que não é justo que outras pessoas do mesmo sexo que o seu sejam mais magras que você? 123456

26. Já vomitou para se sentir mais magro(a)? 123456

27. Quando acompanhado(a), preocupou-se em ocupar um espaço excessivo (por exemplo, sentado(a) num sofá ou no banco de um transporte público? 123456

28. Preocupou-se com o seu corpo estar com "pneus"? 123456

29. Ver o seu reflexo (por exemplo, num espelho ou na vitrine de uma loja) fez com que se sentisse mal em relação ao seu corpo? 123456

30. Beliscou áreas do seu corpo para ver a quantidade de gordura que existe? 1234 56

31. Evitou situações nas quais as pessoas pudessem ver o seu corpo (por exemplo, vestiários)? 123456

32. Já tomou laxantes para se sentir mais magro(a)? 123456

33. Sentiu-se particularmente desconfortável com a forma do seu corpo, quando na companhia de outras pessoas? 123456

34. A preocupação com a forma do seu corpo fez com que sentisse que deveria fazer exercício físico? 123456 


\section{Anexo 3 - TERMO DE CONSENTIMENTO LIVRE E ESCLARECIDO}

Parecer aprovado pelo CEP no 4.251.794

Convidamos o (a) Sr. (a) para participar do estudo científico AVALIAÇÃO DOS FATORES DE RISCO PARA O DESENVOLVIMENTO DE TRANSTORNOS ALIMENTARES, sob a responsabilidade da pesquisadora Profa. Dra. Dorotheia Schimdt França, cuja pesquisa pretende avaliar os fatores de risco para o desenvolvimento de transtornos alimentares em estudantes de uma instituição privada do norte de Minas Gerais.

A sua participação é voluntária e se dará por meio de seleção aleatória de participantes correspondentes a população estudada, que terão de responder questionários validados autoaplicáveis, o Body Shape Questionnaire (BSQ) e o Eating Attitudes Test (EAT-26), com perguntas relacionadas ao comportamento alimentar e imagem corporal. Também será necessário fornecer variáveis sociodemográficas e dados antropométricos.

Os riscos decorrentes de sua participação neste estudo são a possibilidade de constranger-se frente às perguntas dos questionários, caso isso ocorra, o procedimento pode ser interrompido ou suspenso. Devido ao fornecimento de informações pessoais, há o risco de exposição destas, o que será minimizado pelos pesquisadores, evitandose ao máximo a quebra do sigilo e anonimato dos participantes.

Se o(a) Sr.(a) aceitar participar, estará contribuindo para uma análise epidemiológica do risco de desenvolvimento de transtornos alimentares, podendo trazer benefícios, como desenvolvimento de estratégias de prevenção, para a população vulnerável. Se após consentir em sua participação o(a) Sr.(a) desistir de continuar participando do estudo, poderá retirar o seu consentimento em qualquer fase da pesquisa, independentemente do motivo, o que não resultará qualquer prejuízo a sua pessoa.

O(a) Sr.(a) não terá nenhuma despesa e também não receberá qualquer remuneração pela participação neste estudo.

Os dados obtidos da pesquisa serão objeto de análise e publicação, mas a sua identidade não será divulgada, sendo preservada em sigilo.

Para qualquer outra informação, o(a) Sr.(a) poderá entrar em contato com a pesquisadora pelo telefone (38)

, ou poderá entrar em contato com o Comitê de Ética em Pesquisa - CEP das Faculdades Integradas Pitágoras de Montes Claros, situado à rua Aida Mainartina, número 80, bairro Ibituruna, telefone (38)3214-7100, ramal 205, cidade de Montes Claros, Minas Gerais. 


\section{CONSENTIMENTO PÓS-INFORMAÇÃO}

$\mathrm{Eu}$ fui informado (a) sobre os objetivos do estudo científico pelo seu responsável e qual será a minha participação. Declaro ter entendido perfeitamente as explicações do pesquisador. Por isso, declaro consentir em participar do estudo científico, e concordo com as condições estabelecidas acima explicitadas. Este documento será emitido em duas vias assinadas por mim e pelo responsável pela pesquisa, cabendo uma via a cada um.

Montes Claros,

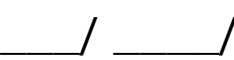

Assinatura do participante

(Impressão do dedo polegar, se for o caso)

Assinatura do Pesquisador Responsável 


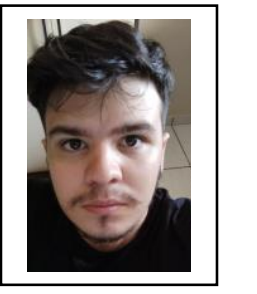

Leonardo Bruno Melo Reis

$\underline{\text { ORCID Lattes }}$

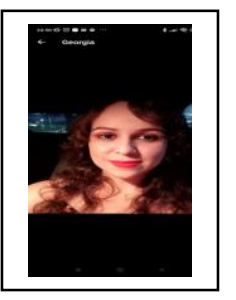

Georgia Guimarães de Castro

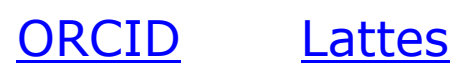

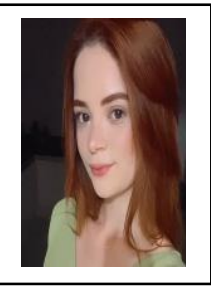

Larissa Rafaella Pereira Tôrres

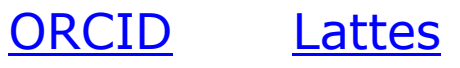

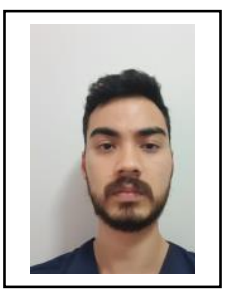

Rodrigo Satoshi Oda Santos

$\underline{\text { ORCID Lattes }}$

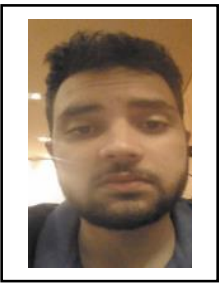

João Vitor Costa

$\underline{\text { ORCID Lattes }}$

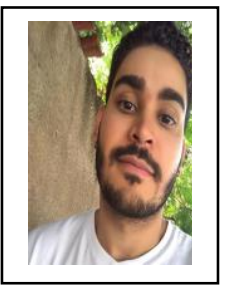

Matheus Gomes Pereira

$\underline{\text { ORCID Lattes }}$

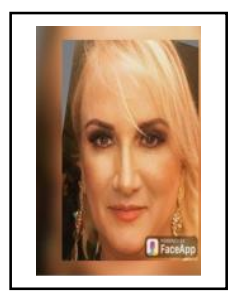

Dorothéa Schmid França

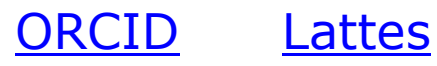

